\title{
Antidepressant drugs promote the heterodimerization of the dopamine D2 and somatostatin Sst5 receptors - fluorescence in vitro studies
}

\author{
Kinga Szafran¹, Sylwia Łukasiewicz², Agata Faron-Górecka1, \\ Magdalena Kolasa ${ }^{1}$, Maciej Kuśmider ${ }^{1}$, Joanna Solich ${ }^{1}$, \\ Marta Dziedzicka-Wasylewska ${ }^{1,2}$ \\ ${ }^{1}$ Department of Pharmacology, Institute of Phar macology, Polish Academy of Sciences, Smętna 12, \\ PL 31-343 Kraków, Poland \\ ${ }^{2}$ Department of Physical Biochemistry, Faculty of Biochemistry, Biophysics and Biotechnology, \\ Jagiellonian University, Gronostajowa 7, PL 30-387 Kraków, Poland
}

Correspondence: Marta Dziedzicka-Wasylewska, e-mail: marta.wasylewska@gmail.com

\begin{abstract}
:
Background: The interaction between the dopaminergic and somatostatinergic systems and their role in mood regulation have been well-documented. Therefore, we decided to investigate the effect of antidepressant drugs on the heterodimerization of the dopamine D2 and somatostatin Sst5 receptors.

Methods: The human receptor proteins were tagged with fluorescent proteins, expressed in the HEK 293 cells and incubated with antidepressant drugs: desipramine and citalopram. To determine the FRET efficiency, the fluorescence resonance energy transfer (FRET) and photobleaching confocal microscopy techniques were used.

Results: We found that the efficiency of FRET is markedly increased in cells coexpressing the somatostatin Sst5 and dopamine D2 receptors after $48 \mathrm{~h}$ of incubation with desipramine and citalopram.

Conclusions: In the present study we provide physical evidence, based on FRET analysis, that antidepressants increase Sst5 and D2 receptors heterodimerization. The effect is specific because desipramine in the incubation medium uncouples other pairs of receptors, such as the dopamine D1-D2 receptors.
\end{abstract}

Key words:

somatostatin receptor 5, dopamine receptor 2, antidepressants, FRET, heterodimerization

\begin{abstract}
Abbreviations: CFP - cyan fluorescent protein, CIT - citalopram, D2 - dopamine type 2 receptor, DMI - desipramine, FRET - Förster (Fluorescence) Resonance Energy Transfer, GPCR - G-protein-coupled receptors, HEK 293 - Human Embryonic Kidney 293 cell line, pbFRET - photobleaching FRET, Sst5 - somatostatin type 5 receptor, YFP - yellow fluorescent protein
\end{abstract}

\section{Introduction}

Dopaminergic and somatostatinergic system interactions have been suggested for many years based on anatomical, behavioral and biochemical studies. It has 
been reported that dopamine administration regulates somatostatin release in the rat striatum and hippocampus [17] and that selective dopamine D1 and D2 receptor agonists increase somatostatin receptor density in the striatum [8]. Likewise, somatostatin positively modulates dopamine release in the striatum [3, 19]. Dopamine and somatostatin have also been implicated in the pathophysiology of depression because of their potential roles in mood regulation. Reduced levels of somatostatin have been observed in the cerebrospinal fluid $[10,18]$ and recently in the subgenual anterior cingulate cortex [20] of depressive patients. Intracerebroventricular administration of somatostatin results in an antidepressant-like effect in rats, as shown by a forced swim test [6]. Furthermore, chronic desipramine treatment selectively potentiates somatostatin-induced dopamine release in the nucleus accumbens and the striatum [12]. It has also been shown that chronic administration of antidepressants influences somatostatin levels and somatostatin receptor density in rat brains [13]. More recent studies show that imipramine upregulates somatostatin release in the mouse hypothalamus, eliciting antidepressantlike effects in tail suspension test [11].

The molecular basis of this functional interaction between the somatostatinergic and dopaminergic systems may stem from the interaction of the somatostatin and dopamine receptors. These receptors were found to share some similarities: they are members of the $\mathrm{G}$ protein-coupled receptors (GPCRs) family, show sequence homology and appear to be structurally related [6]. It has been demonstrated that members of both superfamilies, when coexpressed in the same cell and in the presence of specific ligands, may interact at the membrane level, forming homo- and heterodimers. These dimers may constitute a novel receptor that can activate alternative pathways, possibly enhancing ligand affinity and signal transduction [7].

It has been established by immunocytochemical studies that the dopamine D2 and somatostatin Sst5 receptors colocalize in medium-sized aspiny interneurons in the striatum and pyramidal neurons in the cerebral cortex, an observation that suggests the possibility of functional interactions between these receptors. It has been shown that the dopamine D2 and somatostatin Sst5 receptors, when coexpressed in the same cell, undergo ligand-dependent heterodimerization with enhanced functional activity. D2-Sst5 heterodimerization is associated with a modification in ligand binding and a synergistic effect on the activa- tion of the transduction pathway because both receptors signal through the inhibition of adenylyl cyclase via Gi proteins [16].

In the present study, we investigated the heterodimerization of the human D2 and Sst5 receptors upon treatment with antidepressant drugs (desipramine and citalopram) in the proposed in vitro model by employing biophysical approaches, such as photobleaching fluorescence resonance energy transfer microscopy. Förster (Fluorescence) Resonance Energy Transfer (FRET) is a physical phenomenon that is being used more and more in biomedical research and drug discovery, as it allows the study of interactions between proteins in a single cell. FRET is a process that relies on the distance-dependent transfer of energy between an excited donor molecule and an acceptor molecule in a non-radiative way. This technique provides insight into the interactions between proteins that are in close proximity (less than $10 \mathrm{~nm}$ ) to each other, which makes it an excellent method for measuring the extent to which receptors dimerize. One of the established and commonly used techniques for measuring FRET is acceptor photobleaching [14]. It is based on the fact that FRET reduces the amount of fluorescence released by the donor fluorophore. This method involves measuring the donor fluorescence intensity in the same sample before and after destroying the acceptor by photobleaching. If FRET is initially present, an increase in unquenched donor fluorescence occurs.

We used two antidepressant drugs with different pharmacological profiles: desipramine a tricyclic antidepressant that inhibits the continued uptake of noradrenaline and, to a lesser extent, serotonin; and citalopram, a serotonin uptake inhibitor. Although none of the antidepressants used in this study have any affinity for the dopaminergic or somatostatinergic receptors [2], $48 \mathrm{~h}$ of incubation has been found to affect their ability to form heterodimers.

\section{Materials and Methods}

\section{Materials}

HEK293 cells were obtained from the American Type Culture Collection (Manassas, VA, USA). Cell culture reagents were purchased from Gibco (Carlsbad, CA, USA), Sigma-Aldrich (Poznań, Poland) and PAA Laboratories $\mathrm{GmbH}$. Molecular biology reagents were 
obtained from Fermentas (Vilnius, Lithuania). Oligonucleotides were synthesized by IBB PAN (Warszawa, Poland). The pcDNA3.1(+) plasmids encoding human Sst5 and D2 receptors were obtained from the UMRcDNA Resource Center (University of Missouri, Rolla, MO, USA). The pECFP-N1 and pEYFP-N1 vectors were purchased from BD Biosciences, Clontech (Palo Alto, CA, USA). Desipramine and citalopram were obtained from Sigma-Aldrich (Poznań, Poland), and somatostatin-14 was obtained from ProSpec (Rehovot, Israel).

\section{Construction of fusion proteins}

The human somatostatin Sst5 and D2 genes cloned into the pcDNA3.1(+) plasmids were labeled with cDNA encoding enhanced CFP (ECFP) or enhanced YFP (EYFP). Briefly, the cDNAs encoding the D2 and Sst5 receptors were PCR-amplified. The primers used were the forward universal primer for pcDNA3.1 $(+)$ and the reverse primer, which removed the STOP codon and introduced a unique Xho I restriction site. Using Nhe I and Xho I restriction enzymes, the entire coding sequences were inserted into the pECFP-N1 and pEYFP-N1 vectors. Subsequently, receptors tagged with $\mathrm{CFP}$ protein were used as the fluorescence donor, and receptors tagged with YFP were used as fluorescence acceptor.

\section{Cell culture and transfection}

Human Embryonic Kidney 293 (HEK 293) cells were cultured on coverslips at $37^{\circ} \mathrm{C}$ and $5 \% \mathrm{CO}_{2}$ atmosphere in Dulbecco's modified essential medium supplemented with $10 \%$ fetal bovine serum and $1 \% \mathrm{~L}$ glutamine. Using a calcium phosphate precipitation procedure, the cells were transiently co-transfected with the pcDNA3.1 plasmid containing the sequences of the somatostatin Sst5 and dopamine D2 receptors tagged with fluorescent proteins (CFP and YFP). At $48 \mathrm{~h}$ after transfection, the cells were incubated with somatostatin-14 $(1 \mu \mathrm{M})$ for $30 \mathrm{~min}$ or desipramine and citalopram (10, 1 and $0.1 \mu \mathrm{M})$ for 24,48 and $72 \mathrm{~h}$, and fixed with a $4 \%$ paraformaldehyde solution for $15 \mathrm{~min}$ at room temperature. Then, the cells were washed, mounted onto slides and processed for confocal microscopy FRET measurement.

\section{Confocal microscopy - FRET measurement}

Confocal laser scanning microscopy was performed using a Leica TCS SP2 microscope (Leica Microsystem, Mannheim, Germany). FRET was measured with the Acceptor Photobleaching application using Leica Software as follows: images in the CFP (donor) and the YFP (acceptor) channel were acquired before photobleaching. A region of interest was selected, and the acceptor (YFP) was photobleached by scanning repeatedly with the $514 \mathrm{~nm}$ laser line until the fluorescence signal was at the $50 \%$ level. Then, postphotobleached images for CFP and YFP were acquired by scanning with the appropriate laser line. The FRET efficiency was calculated from the following equation:

$$
\mathrm{FRET}_{\text {eff }}=\left(\mathrm{D}_{\text {post }}-\mathrm{D}_{\text {pre }}\right) / \mathrm{D}_{\text {post }}
$$

where $D_{\text {post }}$ is the fluorescence intensity of the donor after acceptor photobleaching, and $\mathrm{D}_{\text {pre }}$ is the fluorescence intensity of the donor before acceptor photobleaching. The FRET efficiency is considered to be positive when $\mathrm{D}_{\text {post }}>\mathrm{D}_{\text {pre }}$.

\section{Statistical analysis}

The obtained data are presented as the mean \pm SEM. Statistical significance was evaluated using the $t$-test or by a one-way ANOVA, followed by a Dunnett's test for post-hoc comparisons. For the statistical analysis of the data, GraphPad Prism version 5.00 for Windows (GraphPad Software) was used.

\section{Results and Discussion}

To investigate the interaction between the analyzed receptors, we transiently co-transfected HEK 293 cells with dopamine D2 and somatostatin Sst5 receptors tagged with fluorescent proteins (CFP, YFP). Confocal microscopy observations indicated that both dopamine D2 and somatostatin Sst5 receptors colocalize at the plasma membrane (Fig. 1).

First, the agonist-induced formation of heterodimers was investigated. After $48 \mathrm{~h}$ of transfection, cells were treated with somatostatin-14 (1 $\mu \mathrm{M})$ for $30 \mathrm{~min}$ (according to [16]). The observed FRET efficiency was markedly increased compared to the non- 

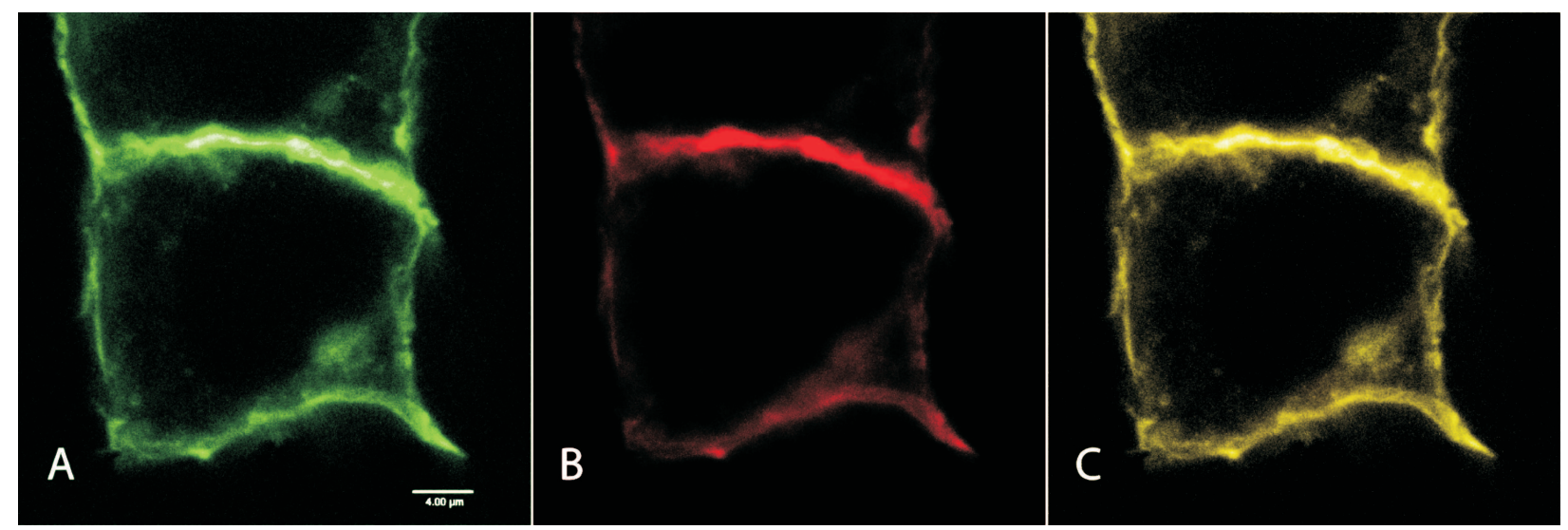

Fig. 1. Colocalization of fluorescently tagged somatostatin Sst5 and dopamine D2 receptors on the membrane surface of transiently transfected HEK 293 cells. Sst5-CFP receptor is shown in green (A) and D2-YFP receptor is shown in red (B). Coexpression of Sst5 and D2 receptors can be seen by the yellow color in the merged image (C)

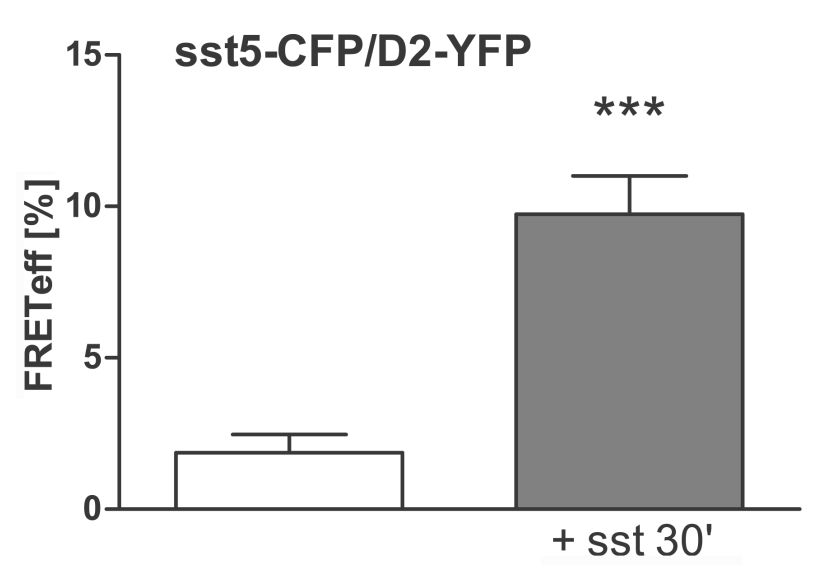

Fig. 2. Somatostatin stimulation of HEK293 cells coexpressing sst5CFP (fluorescence donor) and D2-YFP (fluorescence acceptor) receptors. Somatostatin-14 $(1 \mu \mathrm{M})$ was present in the incubation medium for $30 \mathrm{~min}$. FRET efficiency was measured by acceptor photobleaching. The data are presented as the mean \pm SEM. The statistical significance between the means was evaluated by the $t$-test. (Asterisks indicate $p<0.001$ )

treated cells $(9.74 \pm 1.27 \%$ vs. $1.83 \pm 0.59 \%$, respectively, $\mathrm{p}<0.001$ ) (Fig. 2). This result confirms previous reports that the heterodimerization of the somatostatin Sst5 and dopamine D2 receptors is induced by agonist stimulation and that in the absence of ligand, no significant pre-formed heterodimers exist [6].

We then investigated the effect of antidepressant drugs upon the dimerization of the Sst5 and D2 receptors. The co-transfected cells were incubated for 24, 48 and $72 \mathrm{~h}$ with an antidepressant drug - either desipramine or citalopram (Fig. 3).
Desipramine (at concentrations of 10, 1 and $0.1 \mu \mathrm{M}$ ) present in the incubation medium for $48 \mathrm{~h}$ induced a significant increase in FRET efficiency (respectively: $12.7 \pm 2.7 \%, 15.1 \pm 0.9 \%, 11.9 \pm 1.7 \%$ vs. $3.6 \pm 1.2 \%, \mathrm{p}<0.05)$ in the cells coexpressing both the Sst5 and D2 receptors. Similarly, citalopram induced a significant increase in the FRET efficiency that was measured following $48 \mathrm{~h}$ of citalopram being present in the culture medium at concentrations of 10 and $1 \mu \mathrm{M}$ (respectively: $12.4 \pm 1.3 \%$ and $14.6 \pm 1.8 \%$ vs. $3.6 \pm 1.2 \%, \mathrm{p}<0.01)$. No significant changes were observed after 24 or $72 \mathrm{~h}$ of antidepressant presence in the incubation medium.

The obtained data indicate that desipramine and citalopram presence for $48 \mathrm{~h}$ in a culture medium affects the ability of the Sst5 and D2 receptors to form heterodimers, despite the lack of affinity of these drugs for the dopamine or somatostatin receptors [2].

The present study provides the physical evidence, based on FRET analysis, that antidepressants promote dimerization of the Sst5 and D2 receptors. The mechanism of this influence might be for antidepressant drugs to modulate cell membrane fluidity, leading to the enhancement of receptor-receptor interactions. Previous studies have shown that chronic antidepressant treatment can lead to alterations in the lipid membrane, causing the redistribution of proteins and allowing them to be more mobile and therefore able to interact with each other [4]; however, this antidepressant-induced increased mobility of membrane proteins may also cause a disruption of their interactions. On the other hand, in our preliminary stud- 


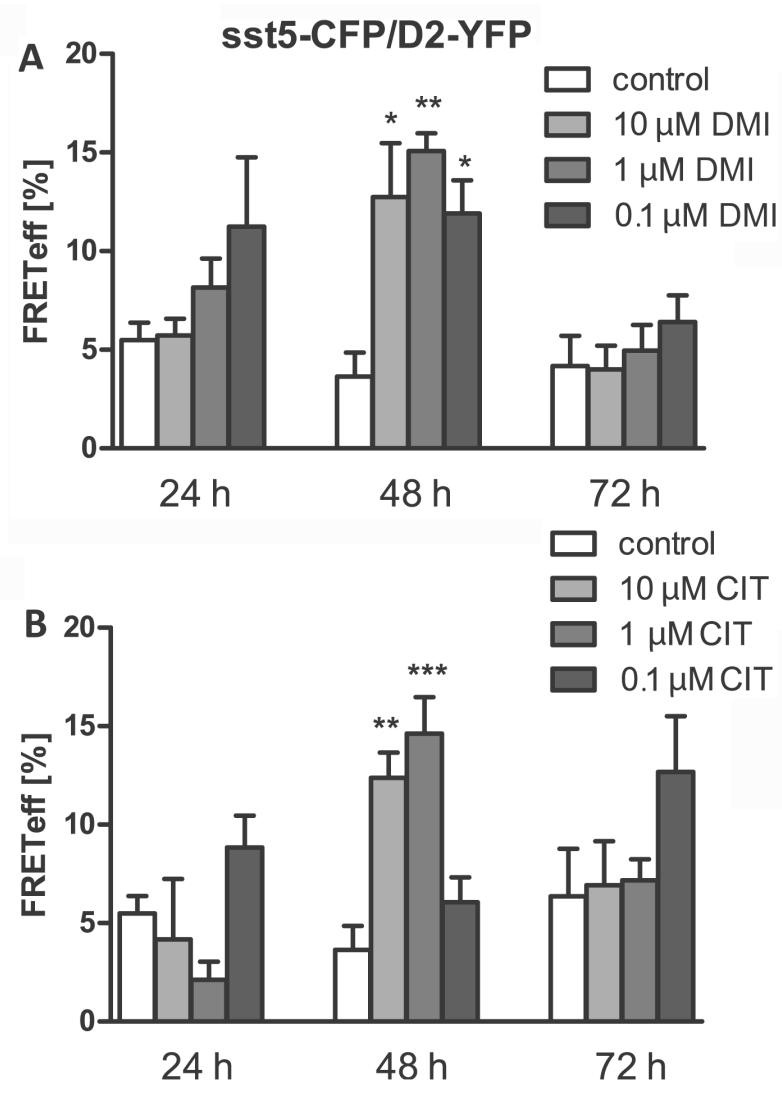

Fig. 3. FRET efficiency of the sst5-CFP and D2-YFP pairs by acceptor photobleaching. The data are presented as the mean \pm SEM. Antidepressant drugs (A - desipramine, $\mathbf{B}$ - citalopram) at concentrations of 10,1 , or $0.1 \mu \mathrm{M}$, were present in the incubation medium for 24,48 and $72 \mathrm{~h}$ prior to cell fixation. The statistical significance was evalu ated using a one-way ANOVA, followed by a Dunnett's test for posthoc comparisons. ( $p<0.05 ;{ }^{\star *} p<0.01 ;{ }^{* * *} p<0.001$ )

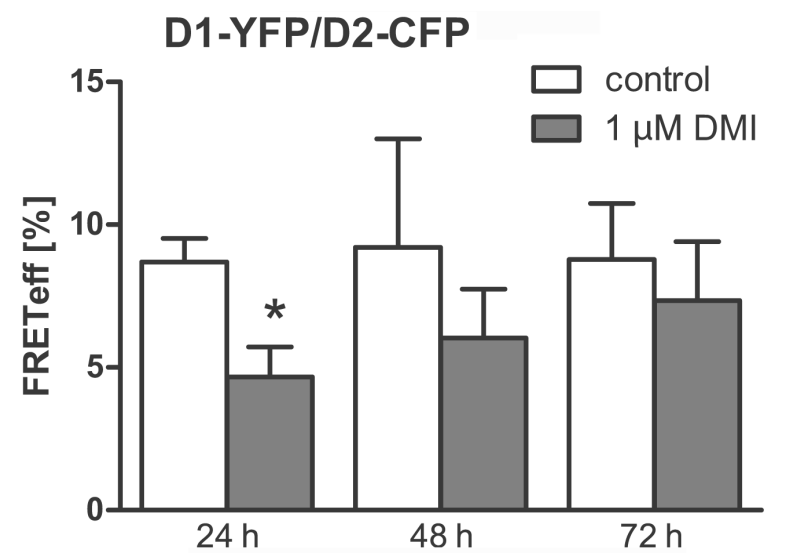

Fig. 4. The effect of desipramine on changes in FRET efficiency in HEK293 cells transiently cotransfected with plasmids containing D1-YFP and D2-CFP fusion proteins. FRET efficiency was measured by acceptor photobleaching. Cells were incubated with desipramine $(1 \mu \mathrm{M})$ for 24,48 and $72 \mathrm{~h}$ prior to cell fixation. The data are presented as the mean \pm SEM. The statistical significance between the means was evaluated by the $t$-test $\left({ }^{*} p<0.05\right)$ ies, we have observed an opposite effect of antidepressants on a different pair of receptors, the dopamine D1-D2 receptors, because a significant reduction in FRET efficiency after $24 \mathrm{~h}$ of incubation with desipramine $(1 \mu \mathrm{M})$ was observed (Fig. 4). These findings are in agreement with the recent studies of Pei et al., which show that the coupling between the dopamine D1 and D2 receptors is markedly increased in the postmortem brains of patients who had suffered from major depression, and that uncoupling the D1-D2 receptor complex exerts an antidepressant-like effect [15]. Therefore, the effect of antidepressant drugs on D2-Sst5 receptor heterodimerization seems to be specific.

In the present study, we validated the use of FRET and photobleaching in the studies of membrane protein dimerization in response to specific ligands and drugs. The high sensitivity of pbFRET is ideally suited for the study of molecular interactions in individual cells. The acceptor photobleaching technique has been widely used to demonstrate the oligomerization of different pairs of receptors $[1,5,16]$. GPCR oligomerization is difficult to analyze in native cells; therefore, human embryonic kidney cell line 293, as well as other cell lines, have been widely used in resonance energy transfer studies of membrane receptors because these cells provide an accepted model in which fluorescently tagged receptor proteins can be efficiently expressed [9]. Although the experiments performed in transfected in vitro models do not represent a physiological state, it might be expected that a similar situation occurs in the membranes of neural cells coexpressing both receptors. However, such conclusions need to be confirmed in vivo.

Numerous biochemical and behavioral studies have reported that dopamine and somatostatin may play a role in the pathophysiology of depression. Somatostatin is known to increase dopaminergic activity in the striatum, although the specific receptors that mediate this effect are still unknown. The results presented in this work may be considered to be evidence for a potential molecular mechanism underlying the antidepressant-like effect of somatostatin, which influences dopamine-mediated behavioral responses. Synergistic effects on the transduction pathway via dimerization of the dopamine D2 and somatostatin Sst5 receptors may enhance the antidepressant effect. As they are distinct from monomers, Sst5-D2 dimers may also activate unknown signal pathways. 
Dopamine D2 and somatostatin Sst5 heterodimers with enhanced functional activity may be considered to be novel mediators of antidepressant mechanisms and may possibly serve as a new drug target in research on the improvement of therapy for depression. However, still more studies are required to elucidate the physiological or pathological consequences of heterodimerization in vivo.

\section{Acknowledgment:}

This study was supported by grant DeMeTer (project number: POIG.01.01.02-12-004/09; 3.6).

\section{References:}

1. Baragli A, Alturaihi H, Watt HL, Abdallah A, Kumar U: Heterooligomerization of human dopamine receptor 2 and somatostatin receptor 2: co-immunoprecipitation and fluorescence resonance energy transfer analysis. Cell Signal, 2007, 19, 2304-2316.

2. Baudry A, Mouillet-Richard S, Launay JM, Kellermann $\mathrm{O}$ : New views on antidepressant action. Curr Opin Neurobiol, 2011, 21, 1-8.

3. Chesselet MF, Reisine TD: Somatostatin regulates dopamine release in rat striatal slices and cat caudate nuclei. J Neurosci, 1983, 3, 232-236.

4. Donati RJ, Thukral C, Rasenick MM: Chronic treatment of C6 glioma cells with antidepressant drugs results in a redistribution of Gso. Mol Pharmacol, 2001, 59, 1426-1432.

5. Dziedzicka-Wasylewska M, Faron-Górecka A, Andrecka J, Polit A, Kuśmider M, Wasylewski Z: Fluorescence studies reveal heterodimerization of dopamine D1 and D2 receptors in the plasma membrane. Biochemistry, 2006, 45, 8751-8759.

6. Engin E, Stellbrink J, Treit D, Dickson CT: Anxiolytic and antidepressant effects of intracerebroventricularly administered somatostatin: behavioral and neurophysiological evidence. Neuroscience, 2008, 157, 666-676.

7. Ferone D: Somatostatin and dopamine receptors. Tumori, 2010, 96, 802-805.

8. Izquierdo-Claros RM, Boyano-Adanez MC, Larsson C, Gustavsson L, Arilla E: Acute effects of D1- and D2receptor agonist and antagonist drugs on somatostatin binding, inhibition of adenylyl cyclase activity and accumulation of inositol 1,4,5-trisphosphate in the rat striatum. Mol Brain Res, 1997, 47, 99-107.

9. Łukasiewicz S, Błasiak E, Faron-Górecka A, Polit A, Tworzydło M, Górecki A, Wasylewski Z, Dziedzicka-
Wasylewska M: Fluorescence studies of homooligomerization of adenosine A2A and serotonin 5-HT1A receptors reveal the specificity of receptor interactions in the plasma membrane. Pharmacol Rep, 2007, 59, 379-392.

10. Molchan SE, Lawlor BA, Hill JL, Martinez RA, Davis CL, Mellow AM, Rubinow DR, Sunderland T: CSF monoamine metabolites and somatostatin in Alzheimer's disease and major depression. Biol Psychiatry, 1991, 29, 1110-1118.

11. Nilsson A, Stroth N, Zhang X, Qi H, Falth M, Sköld K, Hoyer D et al.: Neuropeptidomics of mouse hypothalamus after imipramine treatment reveal somatostatin as a potential mediator of antidepressant effects. Neuropharmacology, 2012, 62, 347-357.

12. Pallis E, Thermos K, Spyraki C: Chronic desipramine treatment selectively potentiates somatostatin-induced dopamine release in the nucleus accumbens. Eur J Neurosci, 2001, 14, 763-767.

13. Pallis E, Vasilaki A, Fehlmann D, Kastellakis A, Hoyer D, Spyraki C, Thermos K: Antidepressants influence somatostatin levels and receptor pharmacology in brain. Neuropsychopharmacology, 2009, 34, 952-963.

14. Patel RC, Lange DC, Patel YC: Photobleaching fluorescence resonance energy transfer reveals ligand-induced oligomer formation of human somatostatin receptor subtypes. Methods, 2002, 27, 340-348.

15. Pei L, Li S, Wang M, Diwan M, Anisman H, Fletcher PJ, Nobrega JN, Liu F: Uncoupling the dopamine D1-D2 receptor complex exerts antidepressant-like effects. Nat Med, 2010, 16, 1393-1395.

16. Rocheville M, Lange DC, Kumar U, Patel SC, Patel RC, Patel YC: Receptors for dopamine and somatostatin: formation of hetero-oligomers with enhanced functional activity. Science, 2000, 288, 154-157.

17. Rodriguez-Sanchez MN, Puebla L, Lopez-Sanudo S, Rodriguez-Martin E, Martin-Espinosa A, RodriguezPena MS, Juarranz MG, Arilla E: Dopamine enhances somatostatin receptor-mediated inhibition of adenylate cyclase in rat striatum and hippocampus. J Neurosci Res, 1997, 48, 238-248.

18. Rubinow DR: Cerebrospinal fluid somatostatin and psychiatric illness. Biol Psychiatry, 1986, 21, 341-365.

19. Thermos K, Radke J, Kastellakis A, Anagnostakis Y, Spyraki C: Dopamine-somatostatin interactions in the rat striatum: an in vivo microdialysis study. Synapse, 1996, 22, 209-216.

20. Tripp A, Kota RS, Lewis DA, Sibille E: Reduced somatostatin in subgenual anterior cingulate cortex in major depression. Neurobiol Dis, 2011, 42, 116-124.

Received: December 14, 2011; in the revised form: May 9, 2012; accepted: May 22, 2012. 